\title{
Revuelta, Feminismo y Nueva Constitución en Chile
}

\author{
Revolt, Feminism and the \\ New Constitution in Chile
}

Alejandra Castillo ${ }^{1}$

Cómo citar este artículo: Castillo, A. (2021). Revuelta, feminismo y Nueva Constitución en Chile. Revista de Ciencias Sociales Ambos Mundos, (2), 47-53. https://doi.org/10.14198/ambos.20999

\section{Resumen}

Este artículo propone la revuelta feminista de mayo del año 2018 como antecedente fundamental del Estallido social del mes de octubre del año 2019. La revuelta feminista pondrá en evidencia los modos en que se anudan en Chile androcentrismo, autoritarismo y neoliberalismo. En este artículo pondré atención a la crítica feminista a la universidad, a la política tradicional y al orden Constitucional.

Palabras claves: Revuelta feminista; estallido social- Androcentrismo; Constitución.

\section{Abstract}

This article proposes the feminist revolt of May 2018 as a fundamental antecedent of the social outbreak of October 2019. The feminist revolt will expose the ways in which androcentrism, authoritarianism and neoliberalism are tied together in Chile. In this article I will pay attention to the feminist critique of the university, traditional politics and the Constitutional order.

1. Alejandra Castillo, Universidad Metropolitana de Ciencias de la Educación, Chile. alejandrabcastillov@gmail.com https://orcid.org/0000-00015954-1897 


\section{A MODO DE INTRODUCCIÓN: CONTRA EL NEOLIBERALISMO}

El estallido social del año 2019 se inicia con el lema "evadir no pagar, otra forma de luchar" lanzado por las y los estudiantes de enseñanza media, mientras invitan a quien quiera a utilizar el Metro evadiendo el pago. La revuelta acontece el día viernes 18 de octubre del año 2019. Esta singular protesta por el alza del trasporte público pronto pone en evidencia el peso cotidiano de vivir en un orden neoliberal en el que la mayoría se empobrece y vive en condiciones muy duras.

"Evadir no pagar, otra forma de luchar" logra articular, así, un importante número de demandas no escuchadas en los últimos treinta años: la salud y educación pública, las pensiones, precariedad laboral, bajos sueldos, endeudamiento, la privatización ominosa de los recursos naturales, el agua, por ejemplo. "Evadir no pagar, otra forma de luchar" se vuelve también el 'significante vacío' que encadena las luchas y protestas de los últimos años: la "Revolución pingüina", protesta de las y los estudiantes secundarios por una educación pública y de calidad (2006); la protesta de las y los estudiantes universitarios y secundarios por el "fin del lucro" en la educación (2011); Movimiento No más AFP (2013); Revuelta feminista (2018); y Paro docente (2019).

Si esta revuelta contra el neoliberalismo tiene algún antecedente, éste es la revuelta feminista de mayo del año justamente anterior. Lejos de plantearse desde una petición sectorial o de interés de grupo, el movimiento feminista volvió visible la violencia patriarcal en la silenciosa inercia de las instituciones, en la cotidianidad de la vida privada y en el daño que produce el modelo económico neoliberal en el cuerpo de la sociedad en su conjunto.

Contra el pronóstico del desencanto y la apatía neoliberal, la política en Chile recobró, del tal modo, un olvidado radicalismo de la mano de un feminismo lejano de las moderadas políticas de mujeres de las cuales tuvimos noticia con la vuelta de la democracia a partir de los años noventa. El feminismo se tomó las universidades y el espacio público (Zerán, 2018). Por casi dos meses fuimos parte de la vorágine de la revuelta feminista. Los medios de comunicación se hicieron presente con despachos diarios, reportajes de toda índole que buscaban mostrar el mundo de las "mujeres". En las universidades, a pesar de las tomas, se organizaron innumerables charlas en los campus. Y, por primera vez, luego de muchos años, el feminismo apareció en foros y conversaciones en centros comunales y regionales, en organizaciones sindicales y hasta en los partidos políticos.

La revuelta feminista hizo escuchar muy fuerte un "no más" a los abusos del Estado autoritario y patriarcal. Un "no más" que se vuelve a escuchar partir del 18 de octubre del año 2019 (Castillo, 2020).

En este artículo intento explorar tres momentos de la revuelta feminista chilena que toma lugar en mayo del año 2018. Estos momentos son el problema del acoso en la universidad y su institucionalidad 'androcéntrica', la alteración de la política tradicional por el feminismo y el cuestionamiento del cuerpo de la república masculina. Cabe indicar que estos momentos de cuestionamientos al orden establecido son antecedentes claves del estallido social del año 2019 y la consecuente demanda por una Nueva Constitución.

\section{CONTRA LA UNIVERSIDAD}

La revuelta es una agitación, un desorden. La revuelta, en su movimiento, trastoca órdenes y sentidos. Lo que estaba de un lado queda del otro, lo que estaba oculto se vuelve visible. Si la revuelta es feminista, el orden trastocado es el patriarcal, volviendo visible los modos en que las instituciones reproducen un sistema social 'androcéntrico'.

Así ha ocurrido en Chile en mayo del 2018. Esta revuelta feminista ha tomado lugar, principalmente, en las universidades tanto públicas como privadas. Esta revuelta no solo ha tomado lugar sino que se ha tomado el lugar. De manera inédita para la protesta feminista, esta revuelta de mayo ha optado como estrategia política de la "toma". Entonces, esta revuelta feminista agita el espacio de lo "en común" de manera doble. Primero, interpela a las instituciones universitarias en lo que éstas inercialmente reproducen al patriarcado en las forma del acoso sexual y la educación sexista; y segundo, interviene la propia lógica de la protesta política al volver claro que su cuerpo no ha sido otro que el de la universalidad masculina. La revuelta feminista, entonces, insospechadamente se toma, al mismo tiempo, el lugar de la universidad y la política de la subversión.

1. Por androcentrismo entiendo una ordenación político social en cuyo centro está la figuración masculina en cuanto a la transmisión de experiencias, la expresión de afectos y la formulación de saberes. 
Tras la agitación de la revuelta feminista se evidencia la urgencia de los cambios para transformar el androcentrismo que la universidad promueve $-y$ que despliega en la sociedad toda. Tales cambios tendrían que ir en dirección de posibilitar institucionalmente políticas paritarias en la contratación tanto de docentes como de personal administrativo. Establecer listas paritarias para la elección de cargos de dirección en todas las instancias. En el nivel de las carreras, escuelas o departamentos se deberían incorporar líneas de trabajo e investigación feminista (en el amplio sentido de la palabra) que deslean crítica e intencionadamente el corpus textual que las disciplinas portan.

Estos cambios tendrían que repensar el vínculo entre universidad, docencia e investigación. Toda vez que la formación universitaria no está desconectada de los modos en que se hace investigación que, como sabemos hoy, se describe en términos de productividad, impacto y competencia. Su formato es el paper y su modo de circulación es la indexación, nombre para la acumulación y venta de datos. Si queremos cambiar el signo androcéntrico que la universidad promueve: ¿no tendríamos que cuestionar, y profundamente, este remozado "positivismo" del modelo de producción de conocimiento actual? Modo de acumulación rapaz que asocia capitalismo de plataformas en la producción de conocimientos -completamente separados de lo público político- bajo las figuras de la excelencia y el mérito escondiendo precariedad laboral, subcontratación, sueldos unidos la ejecución de proyectos y despidos eufemísticamente nombrados como "fin de proyecto", "ajustes" o "reestructuración".

Es por lo anterior que transformar el signo androcéntrico con el que se crean y reproducen conocimientos en la universidad actual no implica solo la creación de oficinas de transversalidad de género que de tan arriba en la estructura de las universidades no llegan a enterarse de lo que ocurre en las disciplinas. Tampoco la transformación del androcentrismo ocurrirá solo con la incorporación de un curso obligatorio de "género" en cada una de las mallas disciplinares; un curso de género que de tan general y poco intencionado, en cuanto a los saberes que las disciplinas portan, que puede terminar siendo la mejor respuesta en términos de una "medida" curricular, pero ineficaz en vistas de la transformación del androcentrismo (Castillo, 2018).

Las dos medidas anteriores dejan intactas las perspectivas con las que el saber universitario se presenta como un saber "universalista" y, por ello, lejano de la diferencia de los sexos. Sin embargo, lo sabemos, en esa abstracción universalista se sigue reproduciendo un orden donde hay un solo sexo, el masculino. De ahí que repensar la universidad pase por (im)pensar sus perspectivas, categorías, cánones y formatos de publicación entre otros muchos aspectos.

Es por lo anterior que me gustaría afirmar que la "letra" nunca es secundaria o parasitaria a un orden de dominio. Si lo que buscamos es interrumpir el androcentrismo que la universidad promueve es necesario cambiar el modo en que entendemos y describimos la "política" (su tiempo, su cuerpo, sus urgencias) y los modos en que ésta "escribe" su historia (Kirkwood, 2017).

Para pensar la universidad desdibujando los márgenes del androcentrismo se debe escribir otra historia, otras historias. La "Nueva Historia" o "Historia del Bajo Pueblo" - hoy en crisis- en mucho se quedó en la simple inversión de las categorías del orden historiográfico dominante - sin cuestionamiento-y en la afirmación del pueblo casi siempre figurado en las historias, gestas y cuerpos de los hombres. Bien podría ser dicho, sin embargo, que la historia social de las mujeres es la distancia polémica a esa figuración masculina en la propia historia del bajo pueblo. Lo que había silenciado la historia del bajo pueblo es puesto en escena por la historia de las mujeres. Sí, es cierto, pero nuevamente las categorías que organizan el saber que la Historia porta quedan intactas. La operación historiográfica que describe a la historia de las mujeres no parece ser distinta a la "inversión de la inversión". No olvidemos que el feminismo -que es interrupción y no inversión- habitualmente es percibido como pequeño burgués por las historias del bajo pueblo. Tal vez el tiempo de la revuelta sea también el tiempo justo para (im)pensar la historia, sus historiografías.

\section{CONTRA LA POLÍTICA TRADICIONAL}

En parte a un cálculo y en parte a la historia del movimiento estudiantil, la fecha de inicio de las movilizaciones en las universidades en Chile es el mes de mayo. Es habitual que las movilizaciones sean lideradas por las federaciones de estudiantes que si bien representan al conjunto de la universidad también representan a sectores políticos determinados. Es habitual también que la política, a pesar de cuotas, cupos y discriminación positiva, siga siendo un terreno de hombres. Es habitual, 
por último, que las estrategias estudiantiles vayan en la siguiente progresión: demanda, paralización y toma. Ese mayo del 2018 fue distinto.

A cincuenta años del mayo del 68 francés, esta revuelta estudiantil fue feminista. La conducción de la movilización fue liderada por mujeres quienes se declararon feministas. No habría que pensar en una revuelta feminista cuyo signo sería la irrupción espontánea. Una pequeña historia de la revuelta estudiantil nos indicaría que durante el año 2006 se organiza la primera gran movilización estudiantil exigiendo el fin de la educación de clases. Algunos puntos centrales del petitorio de esa primera protesta nacional estudiantil fueron la derogación de la Ley Orgánica Constitucional de Enseñanza (LOCE), el fin de la municipalización de la educación y un pase de transporte escolar gratuito y unificado. Con excepción del primer punto, el resto no es escuchado por el gobierno de turno, ni por los que le siguieron. El estallido social de octubre del año 2019 tiene su inicio, precisamente, en el alza de la tarifa del pasaje escolar. Entre los años 2019 y el 2006 es posible fechar la protesta de estudiantes de enseñanza media del año 2008, que nos deja la impresionante imagen de la estudiante secundaria María Música Sepúlveda arrojándole el agua de un jarro a la Ministra de Educación de aquél entonces. En ese mismo lapso de tiempo, no es posible olvidar la protesta estudiantil del 2011, que poco a poco se va transformando en protesta social. En octubre de ese año, estudiantes de enseñanza media, junto a dirigentes sociales y apoderadas, se toman la sala de reuniones del ex-Congreso en Santiago mientras sesionaba el Ministro de Educación junto a parlamentarias y parlamentarios. En esa intervención, más que toma, se exige "plebiscito" y que el poder vuelva al pueblo. Ya para esos años se comenzaba a hablar de asambleas constituyentes siguiendo la pista de los relatos de la nueva historia social chilena (Salazar, 2009).

De ahí en más, la movilización estudiantil de secundarios y secundarias no se detiene. Su práctica política se vuelve más compleja, más creativa, interrumpiendo su posición de clase desde y con posiciones feministas y disidentes; pensemos, por ejemplo, en la aparición de los colectivos Las putas babilónicas (2012) y Colectivo Lemebel (2013). Ya para el año 2015, claramente, la consigna estudiantil no era solo el fin de la educación de clases, sino también la necesidad de avanzar por una educación no sexista. En este último punto son claves los posicionamientos de las estudiantes de los Li- $\operatorname{ceos} N^{\circ} 1, N^{\circ} 7$ y del Liceo Carmela Carvajal, entre muchos otros. $Y$ clave también son las profesoras y los profesores que comenzaron a interrumpir los currículos escolares con lecturas no previstas para una educación sexista.

Claves son también las escuelas informales de feminismo, género, sexualidades, disidencias y performance que en una inusual torsión -que une los márgenes de la universidad con los márgenes de los liceos- hace del concepto de lo político un concepto complejo; pienso, por ejemplo, en los "Talleres de venéreas" de la performer Hija de Perra, la CUDS (Colectivo Universitario de Disidencia Sexual), el colectivo anarquista feminista La Alzada, la REPROFICH, en su desvío feminista; y también en prácticas artísticas y escénicas como las realizadas por las compañías de teatro La niña horrible, Teatro público y Furia barroca. ¿Con toda esta fuerza política, imaginativamente desbordada, cómo no íbamos a tener una revuelta feminista en mayo del 2018?

Estas formas de organización, articulación y creación política de sujetos no previstos por la república masculina, sin duda, ponen en cuestión tres de las tesis socio-políticas con que la inteligencia crítica narraba la "apatía política" popular -hasta entonces. Brevemente descritas, estas tesis son, en primer lugar, aquella que indicaba que el orden neoliberal no era solo un modo económico sino que describía una subjetividad que hacía indistinguible una posición de clase. La prueba que se otorgaba para sostener dicha tesis era que la distribución del "voto" en los sectores populares favorecía a partidos políticos de derecha. El problema de esta primera tesis era el olvido de la abrumadora "abstención" que caracterizaba cada acto eleccionario desde el retorno de la democracia. En algún sentido, la política estaba ocurriendo en otro lugar, quizás en esas calles y liceos olvidados por el Estado mínimo. La segunda tesis indicaba que la integración vía consumo volvía irrelevantes la lucha por los derechos y la justicia; y, en tercer lugar, la bancarización de la vida (deudas de todo tipo) hacían impensable una revuelta social. Dos de los libros que dan marco a estas posiciones son Chile actual. Anatomía de un mito y El consumo me consume, ambos escritos por Tomás Moulian (Moulian, 1997; 1998).

Si bien estos dos libros son narraciones potentes y claves para entender el Chile de los años noventa y comienzos del 2000, parecen no dar cabida para la emancipación, al igual que las tesis socio-políticas arriba descritas. Avanzando en pa- 
ralelo, estudiantes de enseñanza media y universitaria - sin tener noticias de esa apatía y consumismo que se les suponía- comienzan a organizarse políticamente, en esa organización el feminismo no estuvo ausente. La revuelta feminista del año 2018 no se detiene y en octubre del 2019 se transforma en la más grande manifestación contra el modelo neoliberal.

Debido a la concatenación de actos, paralizaciones, tomas, marchas, libros, performances, acciones de arte y visuales, el reclamo feminista no es un simple "estar en contra". Implica, sobre todo, un acumulado de saberes disidentes que permiten, por un lado, la necesaria imaginación política para la puesta en escena de prácticas no androcéntricas. En este mismo sentido, la revuelta social de octubre de 2019, a pesar de la aparente emergencia, espontánea, pone en marcha un acumulado de saberes que narran otros cuerpos para la política. Uno de esos cuerpos es el feminismo.

\section{CONTRA LA REPÚBLICA MASCULINA}

De algún modo, esta revuelta feminista da cuenta de la transformación de la universidad chilena. La universidad republicana, como sabemos, es masculina en su organización e institucionalidad, no sabe de mujeres a pesar que en esa exclusión prescriba roles, funciones y lugares para cada uno de los sexos. Sin embargo, a partir de los años ochenta con el diseño neoliberal adoptado con la Constitución de 1980, la universidad republicana transforma de manera rápida y eficiente el mérito por excelencia, el trabajo universitario por índices de productividad e impacto y la gratuidad por endeudamiento. Es este marco neoliberal el que acompaña el ingreso masivo de las mujeres chilenas al espacio universitario estatal y privado tanto a nivel del estudiantado como a nivel de docencia. No obstante, este paso desde el republicanismo al neoliberalismo mantiene intacta la organización institucional masculina de la universidad (Rodríguez Freire y Tello, 2012). De ahí que la universidad en Chile sea hoy neoliberal y androcéntrica. Esta institucionalidad masculina es una de las cosas que visibilizó la revuelta feminista que se inicia en el mes de mayo. Desde la perspectiva organizacional debe ser advertido que de las 27 universidades públicas y estatales chilenas, solo tres son dirigidas por mujeres. A partir de este dato -de ningún modo al margen- nos podemos imaginar el grado de invisibilidad del trabajo de las docentes, la dificultad de ellas para acceder a cargos de dirección y, por lo tanto, la permanente reproducción de un orden sexista.

Desde la perspectiva de la reproducción del conocimiento es importante mencionar que los saberes que las diversas disciplinas portan al describirse - una y otra vez- desde la neutralidad y abstracción no hacen sino que traer a escena - una y otra vez- el cuerpo de la universidad que fundara Andrés Bello en 1843: un cuerpo abstracto y universal, pero que se particulariza masculinamente. De alguna manera, el "separatismo feminista" respondió en los mismos términos al "separatismo masculino" que de antiguo ha dirigido a la universidad chilena.

Nadie se quedó al margen del debate que generó el feminismo. Los medios de comunicación masiva, en especial los matinales, intentaron enmarcar el feminismo como "una cosa de mujeres", el viejo y buen feminismo de "todas las mujeres". Una portada de revista de espectáculos y moda no queriendo quedarse atrás, y quizás no entendiendo mucho de lo que se trataba, calzó a cinco hombres del espectáculo con tacones (Revista Caras, 2018).

La empatía hacia el feminismo como "cosas de mujeres" estaba en todas partes y de modos insospechados. En algún momento todas las mujeres, incluso mujeres de derecha sin ninguna vocación por la igualdad, se declaraban alegremente feministas. El propio Gobierno de derecha de Sebastián Piñera se vio forzado a tomar una posición frente a la movilización feminista. Esta posición no tardó en llegar con la "Agenda mujer": un conjunto de 12 puntos con los que se comprometía el Gobierno a mejorar las oportunidades de las mujeres y acabar con todas las discriminaciones que las afectan (Diario La Tercera, 2018). El encuadre de esta propuesta no fue otro que el conservadurismo y el neoliberalismo (Castillo, 2018).

Sin duda, la mejor palabra para nombrar a esta irrupción feminista es la de "revuelta". Sin una coordinación centralizada, el feminismo que se tomó nuestra cotidianidad volvió visible el orden patriarcal que inadvertidamente se reproducía en casi todo ámbito de cosas. Una vez visibilizada una injusticia de índole sexista aparecía otra y luego otra. Sin una dirección única o un programa predefinido, la revuelta feminista actúo a la manera de un movimiento sísmico sin un epicentro claramente establecido, de ahí la agitación y el desorden que conmocionó las estructuras de la universidad a partir de problemas como el acoso sexual, la 
construcción androcéntrica del saber, las genealogías del feminismo chileno y la posibilidad de un feminismo socialista. En no más de dos meses se pusieron en escena dos siglos de debates y disputas de los feminismos locales y metropolitanos, neoliberales y socialistas.

A pesar de la multiplicidad de espacios tocados por la movilización feminista, la revuelta tuvo como detonante la respuesta moderada otorgada por algunas universidades frente a denuncias realizadas contra académicos por acoso sexual. Esta génesis da pistas del accionar de la revuelta feminista. Distinta a otras movilizaciones estudiantiles cuyo signo es la progresión en las estrategias de presión, la revuelta feminista opta por la forma de la ocupación de los planteles universitarios desde el inicio.

De un día para otro en el mes de mayo, las universidades amanecieron "tomadas", la radicalidad del accionar feminista se entiende por la propia génesis de la demanda. Desde hace unos tres o cuatro años atrás algunas universidades en Chile comenzaron a elaborar Protocolos contra el acoso sexual ${ }^{2}$. La aprobación de estos protocolos permitió hablar del acoso y violencia sexual dentro de la universidad y, más importante aún, permitió presentar denuncias. Los procesos que se llevaron a cabo, sin embargo, no cumplieron con las expectativas de las denunciantes: en algunos casos los académicos fueron reubicados en la misma universidad, otras veces fueron amonestados y sancionados con el alejamiento de la docencia por uno o dos meses y en otras ocasiones no fueron sancionados en lo más mínimo. Esta moderación en las sanciones dejó en evidencia que los protocolos, si bien eran muy útiles para explicitar un orden de violencia sexual dentro de los recintos universitarios, no lo eran tanto si eran aplicados en una institucionalidad jurídica que no incorporaba categorialmente la perspectiva de la violencia de género.

Dicho de otro modo, muchos de los protocolos contra el acoso sexual que las universidades en Chile implementaron se enfrentan a reglamentos académicos y estudiantiles que no reconocen la violencia sexual como un tipo de violencia específica y, por tanto, las sanciones fueron inexistentes

2. Tómese el siguiente caso: con fecha de 10 de enero del año 2017 la Universidad de Chile aprueba Protocolo de Actuación ante denuncias sobre acoso sexual, acoso laboral y discriminación arbitraria, Decreto Exento $\mathrm{N}$. 001817, Universidad de Chile, Santiago-Chile. o moderadas. Es importante destacar que la lógica de implementación de los protocolos ocurrió, principalmente, desde "secretarías de género" y, por ello, su impronta estuvo más cercana a las corrientes de las políticas de mujeres liberales e institucionales. La implementación defectuosa de los protocolos contra el acoso sexual, sin embargo, permitió la inesperada emergencia del feminismo en Chile. La lógica de la protesta estudiantil feminista siguió los siguientes momentos: primero, reconocimiento del universalismo de la ley (existencia de los protocolos); segundo, interpelación y presentación de demandas por parte de las estudiantes; tercero, encuentro fallido con la ley y afirmación de la universidad masculina a pesar de los protocolos; y cuarto, constitución de la enunciación y política feminista.

Es en ese último punto donde la política de mujeres liberal e institucional muta en feminismo y radicalidad. Es en ese último momento en que se percibe el daño de ser mujeres en una universidad cuya institucionalidad es profundamente masculina. Es precisamente, ahí, cuando el movimiento estudiantil muta en movimiento feminista. Esta mutación feminista no busca solo hacer protocolos eficientes sino que pide lo imposible: transformar la lógica androcéntrica que inercialmente la universidad reproduce, pero también -y al mismo tiempohacer de la universidad un régimen de igualdad, garantista y de derechos. Entonces, la revuelta feminista exigirá transformar la institucionalidad jurídica de las universidades incorporando la diferencia sexual en una ordenación política económica no neoliberal. Esta exigencia implica, sin duda, transformar la sociedad chilena en su conjunto, poner fin a la República masculina (Castillo, 2021).

\section{A MODO DE CONCLUSIÓN: UNA CONSTITUCIÓN FEMINISTA}

Lo que fue habitual hasta hace un tiempo atrás en las discusiones sobre la democracia en Chile ya no lo es más. Se suele decir que la búsqueda del consenso como bien principal del régimen democrático chileno habría terminado por vaciar a la democracia de todo contenido, dejando en su lugar solo un conjunto de procedimientos eleccionarios enfrentados a la apatía y la baja participación. Sin embargo, nos equivocamos si pensamos que ésta, nuestra democracia, es meramente procedimental (Fernández y Figueroa, 2015). 
El equívoco es pensar a la democracia en abstracción del marco sustantivo que la constituye y la dota de contenidos: la Constitución de 1980. Lo que se ha evidenciado con el paso de los gobiernos de la ex Concertación era el despliegue y puesta en práctica de su orden sustantivo ligado al ideario político-económico neoliberal.

Es por esa ligazón que nuestra democracia no es en absoluto "meramente" procedimental, cada uno de los conceptos que la describen reproducen una sociedad de mercado. ¿Qué decimos con derechos en este contexto? Un conjunto de bienes alcanzables dependiendo de la capacidad adquisitiva de cada quien, ahí está la educación y la salud para demostrarlo. ¿Qué decimos con participación? No otra cosa que acudir de tanto en tanto a las urnas para legitimar un proceso eleccionario. Salir de este equívoco nos obliga darnos a la tarea de proponer otros modos de pensar los contenidos que describen a la democracia; esto implica, sin duda, proponer otras definiciones para una Nueva Constitución, más aun una Constitución feminista.

Una Constitución feminista obligaría a interrogar los propios fundamentos del derecho moderno cuestionando el androcentrismo que le es propio (Brito, 2020). Una Constitución feminista deberá, en tal sentido, desplazar el primer artículo de la Constitución de 1980 que vincula el cuerpo de la nación con el cuerpo de la familia como orden reproductivo, y justo por ello, con el cuerpo de las mujeres, que es representado de manera restringida. Este encadenamiento hace que el cuerpo de las mujeres esté siempre subordinado a los intereses de la nación.

¿Qué lugar ocupan las mujeres en el encadenamiento nación, familia y reproducción? Esta cadena debe ser rota. Un modo es garantizar los derechos de las mujeres. Entre estos derechos se debe incluir el aborto no restringido a las tres actuales causales que fija la ley. Junto a ello, y sin ánimo de establecer jerarquías de urgencia o relevancia, se debe garantizar la participación política de las mujeres en igualdad de condiciones en todas las instancias de decisión pública, así como se debe garantizar la igualdad de salario en el trabajo, reconocer el trabajo doméstico como trabajo remunerado y, junto a ello, avanzar hacia una renta básica universal. Esta lógica de reconocimiento de derechos es al mismo tiempo una lógica de la redistribución, es decir, un razonamiento que se ordena a partir de la igualdad y la participación de las mujeres en política. Elementos mínimos para definir una Constitución feminista.

\section{REFERENCIAS}

Brito, S. (2020). Por una Constitución feminista. Editorial Libros del Pez espiral.

CAstillo, A. (2021). La república masculina y la promesa igualitaria. Editorial Mimesis.

Castillo, A. (2020). La asamblea de los cuerpos. Sangría editora.

CASTILLO, A. (2018). El feminismo en el espejo del neoliberalismo. Antígona Feminista. https://antigonafeminista.wordpress.com/el-feminismo-en-el-espejo-del-neoliberalismo

KIRKWood, J. (2017). Feminarios. Colectivo Communes.

La Tercera (2018, 23 de mayo). Piñera firma instructivo de 12 puntos sobre equidad de género. Diario La Tercera. https://www.latercera.com/ politica/noticia/pinera-firma-instructivo-12-puntos-equidad-genero/176022/

Moulian, T. (1997). Chile actual. Anatomía de un mito. LOM ediciones.

Moulian, T. (1998). El consumo me consume. LOM ediciones.

Revista Caras (2018, 1 de junio). En los zapatos de ellas. Especial hombres. Revista Caras.

Rodríguez, R. y Tello, M. (2012). Descampado. Ensayo sobre las contiendas universitarias. Sangría editora.

SalazAR, G. (2009). Del poder constituyente de asalariados e intelectuales. LOM ediciones.

ZerÁn, F. (2018). Mayo feminista. La rebelión contra el patriarcado. LOM ediciones. 